\title{
State-dependent dissociation of food consumption and maze running in rats
}

\author{
JOHN H. GILL and HAROLD C. NIELSON \\ University of Utah, Salt Lake City, Utah 84112
}

\begin{abstract}
For 45 days, rats were given $30 \mathrm{~min}$ to consume wet mash immediately after they regained their righting reflexes following an IP injection of $25 \mathrm{mg} / \mathrm{kg}$ of sodium pentobarbital. Then they were trained to traverse a maze for a food reward while drugged, while not drugged, or $2 \mathrm{~h}$ before receiving the drug. After 28 days, the maze training continued but drug conditions were switched. The running latencies showed that running for a food reward was state-dependent. Food consumption also showed dissociation when the drugged condition was discontinued either at the start of maze training or during the drug reversal. Food consumption was not changed when the drugged condition was continued or reinstituted. Additional rats that received equal amounts of drug, but were not fed while drugged, showed that the effects on food consumption were related to the drugged-feeding experience and not to drug experience per se. A possible reorganization of sensory-motor integration associated with drugged feeding was discussed.
\end{abstract}

State-dependent phenomena are those in which responses learned in a particular state, e.g., either a drugged state or the normal state, are dependent upon the presence of that state for their performance. The phenomenon is now well known experimentally (Overton, 1974) and includes flexion responses in hammocks (Girden \& Culler, 1937; Pusakulich \& Nielson, 1972) as well as tasks of escape, avoidance, and approach in T-mazes (Overton, 1964), shuttleboxes (Holmgren, 1964), and barpressing situations (Kubena \& Barry, 1969) in animals as diverse as rats, monkeys, cats, dogs, and goldfish. The phenomenon also has been demonstrated in human beings (Goodwin, Powell, Bremer, Hoine, \& Stern, 1969; Madill, 1967; Swanson \& Kinsbourne, 1976).

The most potent agents for producing statedependent effects purportedly are centrally acting drugs, a comprehensive listing of which is provided by Overton (1972). Electroconvulsive shock (ECS) also produces state-dependent effects (DeVietti \& Larson, 1971; Neilson, 1968) as well as interesting motivational effects on habit retention (Robbins \& Meyer, 1970). When Robbins and Meyer administered ECS to abolish retention while animals were performing either of two motivated tasks (either food approach or shock avoidance), the learned response predictably failed to occur when the animal was tested in the motivational condition in which ECS was given. However, when tested under

Supported by Contract Number DADA 17-73-C-3058 from U.S. Army Medical Research and Development Command. The research described in this article adhered to the "Guide for Laboratory Animal Facilities and Care," as promulgated by the Committee on Revision of the Guide for Laboratory Animal Facilities and Care of the Institute of Laboratory Animal Resources, National Academy of Science-National Research Council. Requests for reprints should be addressed to John H. Gill, Department of Psychology, University of Utah, Salt Lake City, Utah 84112. motivational conditions that did not prevail at the time of the ECS, the learned response was performed. Furthermore, the performance of the response was not related to the time interval between the performance of the response and the ECS, only to the motivational condition that had existed when ECS was given.

A possible relationship between state-dependent performances and motivational variables has only rarely been emphasized in state-dependency research (Bindra, Nyman, \& Wise, 1965; Bindra \& Reichert, 1966, 1967). This seems somewhat unusual, since there have been widespread reports of motivational changes following the development of dependence upon drugs that produce state-dependent effects. Collier (1969), especially, following Wikler (1953), has drawn attention to the lessening of hunger, sex, and fear as primary drives consequent to the development of dependence on drugs.

In the present experiment, we have tried to place an emphasis on motivation in state-dependent learning. Specifically, we tried to associate what generally has been regarded an "unlearned" primary motive (food consumption in a food-deprived rat) with a drugged state so that consumption of food would be dependent on the drugged state. To adequately demonstrate such an effect, we reasoned it would be necessary to show that the effect was not dependent upon other traditional "motivating" conditions. Hence, the procedures were designed to assure that the rats were food deprived and used to the feeding schedule, were familiar with the feeding site, were not ill, and had immediate access to a familiar food. Recent theories of motivation (especially Bindra, 1969, 1974) provide some justification for conceiving of motivated behavior as subject to "incentive" learning effects; but we know of no theory of motivation, or of state dependence, that lends itself to prediction of state dependence of food consumption. 
Although we do not try here to present such a theory of motivation, we do try later in the discussion to account for the observed findings in a new statedependency framework.

\section{METHOD}

Subjects

The subjects were 69 adult male Wistar rats that weighed between 260 and $435 \mathrm{~g}$ at the start of the experiment. They were housed individually in wire-mesh cages in a room that was kept at a temperature of $70^{\circ}-72^{\circ} \mathrm{F}$ and on a 12-h light-dark cycle.

\section{Apparatus}

A U-shaped, single alleyway $(70 \times 76 \times 70 \mathrm{~cm})$ was constructed of plywood with cardboard inserts covering the floor. The startbox was on one end of the maze with a guillotine door that gave access to the alleyway. The goalbox was a removable compartment, $30 \times 10 \times 15 \mathrm{~cm}$ (inside dimensions), with a second guillotine door that was closed as the rat entered it from the maze. Running speeds were recorded from stopwatches.

\section{Procedure}

The procedure was composed of food-consumption training, maze training, and drug-reversal training (or maze retraining). During food-consumption training, the rats were divided into nine subgroups of comparable weight. Three of the subgroups (initially eight rats per subgroup) were, on each of 45 days, given 30-min access to a gruel of wet mash in their home cages immediately upon regaining their righting reflexes after they received injections $(25 \mathrm{mg} / \mathrm{kg}$, IP) of sodium pentobarbital (drugged fed group). Another three subgroups were fed $2 \mathrm{~h}$ after they had regained their righting reflexes (postdrugged fed group). A third set of three subgroups of rats (initially seven rats per subgroup) received no experience either with drug injections or scheduled feeding prior to maze training (non-predrugged group) but were food-deprived for $24 \mathrm{~h}$ prior to the start of maze training.

Maze training consisted of one trial each day for 28 days. The rats were oriented toward the alleyway in the startbox prior to opening the guillotine door, and were allowed a maximum of $500 \mathrm{sec}$ to traverse the alleyway to the goalbox before they were gently pushed to the goal. The goalbox was baited with a generous helping of wet mash; and, once in the goalbox, the rats were allowed to eat for a total of $30 \mathrm{~min}$. Individual goalboxes were detached from the maze after the rat had taken a few bites of food. Drug-reversal training began on the day after the 28th day of maze training and was continued for 12 days, with the same procedures followed.

For maze training and drug-reversal training, the three subgroups in each main group differed by when they received the drug in relation to their maze experience. Specifically, druggedmaze/saline-maze rats (DM-SM) ran the maze during maze training immediately after regaining their righting reflexes after an injection $(25 \mathrm{mg} / \mathrm{kg}$, IP) of sodium pentobarbital (DM); and, during the drug-reversal phase, they ran the maze $30 \mathrm{~min}$ after an equivalent volumetric IP injection of saline (SM). Similarly, saline-maze/drugged-maze rats (SM-DM) initially received a saline injection $30 \mathrm{~min}$ prior to running the maze during maze training; and, during drug reversal, they received an injection of sodium pentobarbital $(25 \mathrm{mg} / \mathrm{kg}$, IP) and ran the maze immediately after regaining righting reflexes. Maze-drugged/druggedmaze rats (MD-DM) ran the maze during maze training before any injection, but received sodium pentobarbital $(25 \mathrm{mg} / \mathrm{kg}$, IP) $2 \mathrm{~h}$ after completing the day's maze-running and eating experience. During the drug-reversal phase, these rats received the injection of pentobarbital and ran the maze immediately
Table 1

The Summary of the Subgroup Treatments of the Three Main Groups

\begin{tabular}{ccc}
\hline Drugged Fed & Postdrugged Fed & Nonpredrugged \\
\hline DM-SM & DM-SM & DM-SM \\
SM-DM & SM-DM & SM-DM \\
MD-DM & MD-DM & MD-DM \\
\hline
\end{tabular}

upon regaining their righting reflexes. Table 1 summarizes the three main treatments during food-consumption training and their subgroups for maze training and drug-reversal training.

Daily measures of latencies to traverse the maze, total grams of wet mash consumed, and body weights were taken. Separate analyses of variance were run for each main group of rats (drugged fed, postdrugged fed, and non-predrugged) on each of the dependent measures. Between-subjects' sources in the analyses were for subgroups of rats (DM-SM vs. SM-DM vs. MD-DM), and within-subjects' sources were composed of Maze Training vs. Drug Reversal along with Days (trials) within Maze Training vs. Days within Drug Reversal. Twelve maze-training days were employed in the main analyses; the remaining data revealed no additional effects. The means of the final 3 days of maze training (Days 26-28), for comparative purposes, are included in each graph and are presented in the results when pertinent to the data descriptions. Analysis of variance effects, $t$ tests, and individual mean comparisons by Duncan tests were evaluated at $p=.05$. The t tests were two-tailed.

\section{RESULTS}

During the course of the experiment, five subjects died in the drugged fed group and three died in the postdrugged fed group because of early failures to adapt to the feeding schedule. One rat died in the nonpredrugged group, possibly from illness. Additional rats were eliminated by random selection in order to arrive at an equal number of rats (five) per subgroup.

\section{Maze Training Results}

Figures 1 and 2, respectively, show the mean grams of wet mash consumed and the mean running latencies (absolute) for each group during the first 12 days of maze training and the 12 days of the drug reversal.

Drugged fed group. For drugged fed subjects, the Duncan test that compared means within the Subgroups by Days Within Phases interaction, $F(44,264)=4.15$, showed clear dissociation of food consumption. On the first day of maze training, the DM-SM subjects, for whom the drugged state continued, consumed greater quantities of wet mash than did either the SM-DM or MD-DM subjects, and they showed no changes in food consumption so long as the drugged state continued. Conversely, when the drug injections were discontinued at the start of maze training for the SM-DM rats, and when feeding preceded the drug injections by $2 \mathrm{~h}$ for MD-DM rats, both of these subgroups ate less than their average of the last 3 days before maze training, t SM-DM (4) $=3.17$, t MD-DM $(4)=3.99$, respectively. These subgroups did not differ on the 26-28-day measure. 


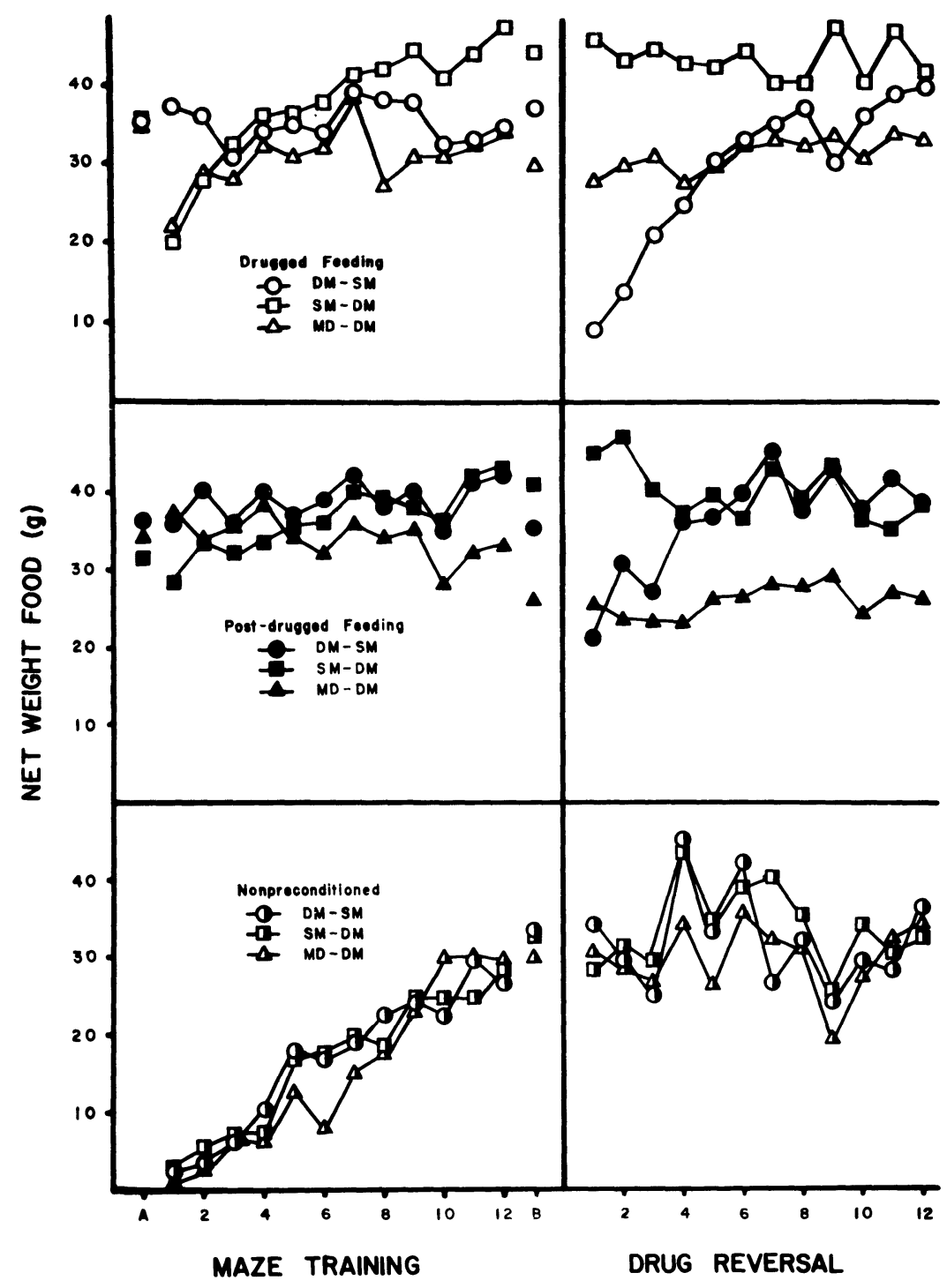

Figure 1. Mean food consumption scores for subgroups across phases.

The Subgroups by Days Within Phases interaction for maze running of drugged fed subjects, $F(44,264)$ $=1.52$, also was evaluated with the Duncan statistic which showed that running speeds generally decreased equally across maze training for each drugged fed subgroup and, except for rats in the MD-DM subgroup, became efficient and stable by Day 4 . For the MD-DM rats, latencies appeared to increase and decrease unsystematically, although they differed from the remaining two subgroups on only 2 of the 12 days and they were not different just before the drug-reversal phase.

Postdrugged fed group. The comparison of means within the Subgroups by Days Within Phases interaction for postdrugged fed subjects, $F(44,264)=3.66$, showed a difference in food consumption between subgroups only on the first day of maze training; the SM-DM rats ate less wet mash than did either of the other two subgroups on that day. However, SM-DM rats did not eat less on the 1 st day of maze training than they themselves had eaten previously $(t=.92)$. This subgroup also increased food consumption across the first few days and stabilized after Day 6.

For maze running, the Subgroups by Days Within Phases interaction, $F(44,264)=1.64$, showed that all postdrugged fed subjects' latencies declined across maze-training trials, were not different from each other, and were fairly stable after Day 5 . However, on Days 26-28, subgroups did differ, $F(1,12)=4.47$, with SM-DM rats showing longer latencies $(\overline{\mathrm{X}}$ absolute $=17.7 \mathrm{sec}$ ) than MD-DM rats $(\overline{\mathrm{X}}$ absolute $=8.0 \mathrm{sec})$. No other differences were observed.

Non-predrugged group. Individual comparisons of means within the Subgroups by Days Within Phases interaction for this group, $F(44,264)=1.70$, showed 


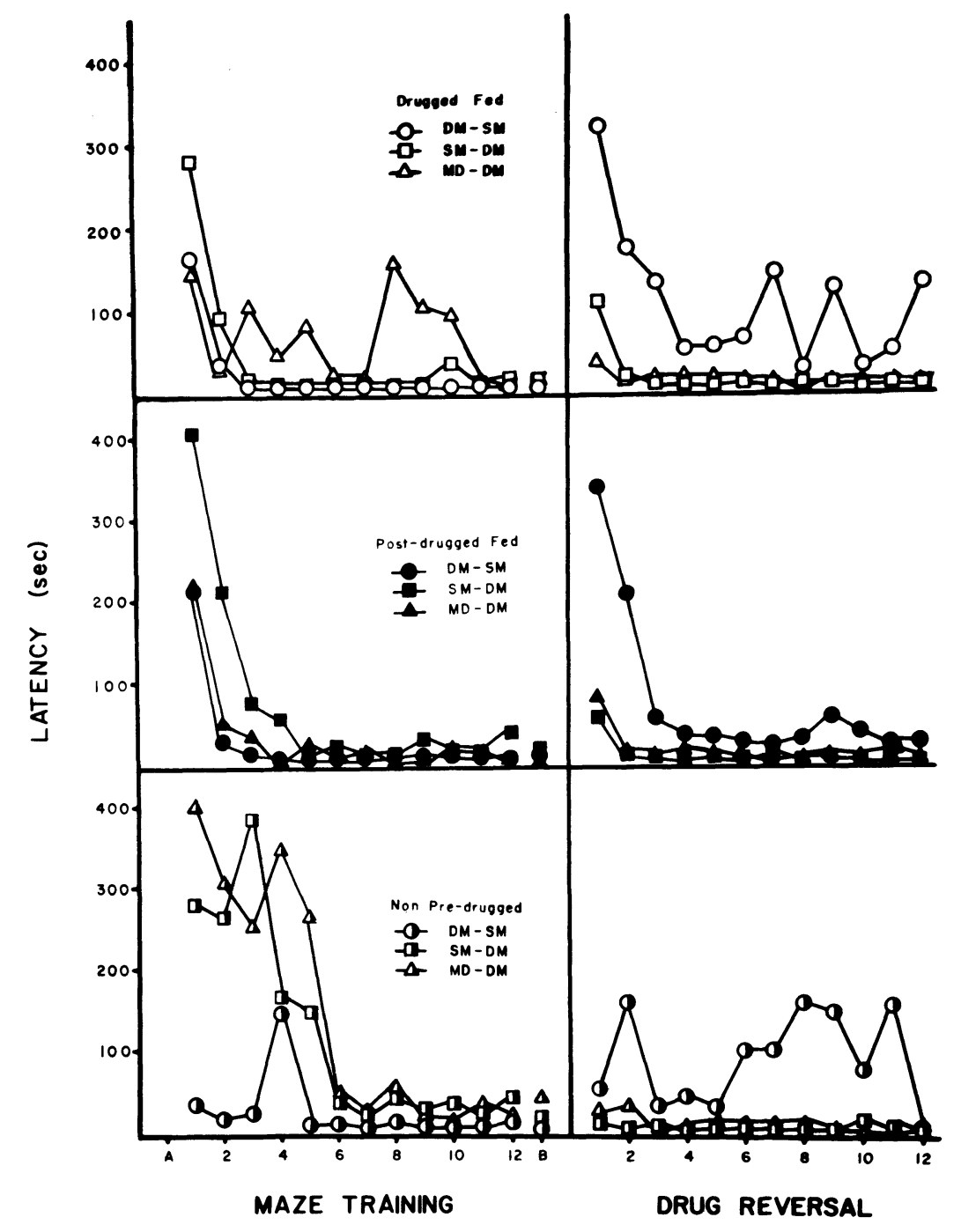

Figure 2. Mean latencies (absolute) for subgroups across phases.

that although food consumption was slight during the first few days of maze training, it increased across days. The subgroups did not differ throughout maze training.

On maze running, the DM-SM subjects in this group showed the shortest latencies, for the first 3 days of maze training, of any of the other subgroups in the experiment. These rats had appeared to bolt from the startbox in a "drunken" fashion at the start of maze training rather than "exploring" the maze as was characteristic of subjects in each of the remaining groups. The rats were observed to eat only scant quantities of food during these early trials. On Day 4, individual comparisons within the Subgroups by Days Within Phases interaction, $F(44,264)=1.88$, showed that the latencies of DM-SM rats increased and these rats then did show signs of exploring the maze. Thereafter, their latencies again were reduced. Subgroups of the non-predrugged group were not different on the final 3 days of maze training.
In summary, there was dissociation of food consumption for drugged fed subjects at the start of maze training when the drug was discontinued for the first time. This effect did not occur for postdrugged fed subjects who had not received feeding in the drugged state or for non-predrugged subjects who had not had previous experience with the drug prior to maze training. The running latencies of each of the subgroups generally reflected normal patterns of learning the maze, except for the DM-SM rats in the non-predrugged group, who, upon receiving the drug for the first time, "bolted" down the maze to the goal. Food consumption and running latencies were stable for all subgroups by the conclusion of 28 days of maze training.

\section{Drug Reversal Results}

Drugged fed group. During the drug reversal, the DM-SM subjects that now were without drug during feeding for the first time since the experiment began 
showed a marked decline in their consumption of food. Specifically, this subgroup ate less than they had eaten on any previous maze-training day. They also ate less than did SM-DM rats on the first 6 days and less than did MD-DM rats on the first 3 days of the drug reversal. Neither SM-DM nor MD-DM rats, which had returned to "drugged feeding," decreased or varied their consumption of food across drug-reversal days.

With drug reversal, the latencies of DM-SM rats increased markedly, showing response dissociation, and their performances remained variable and "disrupted" throughout the drug-reversal phase. The Duncan statistic showed specifically that the latencies of this subgroup were greater on the first day of the drug reversal than were the latencies of SM-DM or MD-DM rats on that day, and than their own latencies had been on any maze-training day except for the 1st day. Also, the DM-SM rats had longer latencies than did SM-DM subjects on 10 of the 12 drug-reversal days and than did MD-DM subjects on 6 of the 12 drug-reversal days. The latencies of both SM-DM rats and MD-DM rats increased without differing only on the 1 st day of the drug reversal. After that, no dissociation of responding could be discerned and their latencies remained stable until the completion of the drug reversal.

Postdrugged fed group. During the first 3 days of the drug reversal, when the drug was withheld from DM-SM rats, they ate less than they had on any maze-training day. However, by the 3rd day of the reversal, their consumption of food increased and remained stable at a level that no longer differed from the level of their food consumption during maze training. The food consumption of SM-DM and MD-DM rats remained unchanged across drug-reversal days.

On the 1st day of the drug reversal, all of the latencies for each subgroup were increased above their final maze-training performances. On that day, a greater increase in latencies was observed for DM-SM rats than for either SM-DM or MD-DM rats, which, themselves, did not differ. The increases appeared to result from both longer emergence latencies and longer running latencies, and, again, are reflective of partial statedependent effects on maze running. In addition, the daily drug-reversal performances after Day 1 showed that the latencies of DM-SM rats remained somewhat increased and were longer than those of SM-DM rats on 7 of 12 and than MD-DM rats on 5 of 12 drugreversal days. The latencies of SM-DM and MD-DM rats were not different throughout the drug reversal.

Non-predrugged group. As during maze training, wet mash consumption did not differ between the subgroups in the non-predrugged group throughout the drug reversal. However, on the 1st day of the drug reversal, there was an increase in mean latencies of DM-SM rats that exceeded their own latencies on Days 5-12 of maze training and exceeded the Day 1 drug-reversal latencies of SM-DM rats, but not of MD-DM rats. DM-SM rats' latencies remained variable throughout the course of the drug reversal and exceeded those of the SM-DM rats on 7 of 12 and of the MD-DM rats on 5 of 12 drugreversal days. This increase demonstrated a moderate state-dependent effect on running for DM-SM rats. However, the latencies of SM-DM and MD-DM subgroups did not increase and were not different on any drugreversal day. Upon receiving the drug for the first time, the latencies of SM-DM subjects remained short. This tendency of rats to bolt down the alleyway when they received the drug for the first time may have minimized any response dissociation that could be expected during drug reversal.

\section{Across-Group, Food-Consumption Comparisons}

In order to compare each subgroup across main groups on their food consumption scores during Day 1 of the drug reversal, three repeated measures analyses of variance were run with Drugged Fed vs. Postdrugged Fed vs. Non-Predrugged groups as the between-subjects' source and the Average of Days 26-28 vs. Day 1 of Drug Reversal as the within-subjects' source. Of primary concern was the determination of a reliable Groups by Days interaction which occurred only in the analysis of DM-SM subgroups, $F(2,12)=23.73$. The Duncan statistic showed that DM-SM subgroups did not differ at the conclusion of maze training; but, on Day 1 of the drug reversal, DM-SM subjects of the drugged fed group consumed less wet mash than did DM-SM subjects of the postdrugged fed group which, in turn, consumed less wet mash than did DM-SM subjects of the nonpredrugged group.

\section{DISCUSSION}

In this experiment, we investigated whether a primary motive associated with food consumption could become state dependent using pentobarbital to induce the drugged state. There were two reasons why we thought such a demonstration would be difficult: (1) generalization across drugged and nondrugged conditions is reported to occur very rapidly (Overton, 1964), and the rats had been feeding in the nondrugged condition before they became experimental subjects, and (2) it is possible that food consumption would be dictated more by food deprivation plus the presence of food than by learning. We also thought that if there were state-dependent effects on food motivation they might be transient, take time to develop, and should not be related to "drug withdrawal." Given these limitations, it was necessary to see a state-dependent effect on food intake for drugged fed DM-SM animals during the maze retraining phase of the experiment, when the rats were switched for the first time to the nondrugged condition; and this occurred. Food consumption was markedly reduced for this group of rats, as was their maze-running efficiency. We also expected SM-DM and MD-DM 
animals of the drugged fed group to eat less when maze training began because food intake and the drugged state then were separated. Both of these subgroups ate less than did DM-SM animals on the first day of maze training, and less than they, themselves, had eaten before maze training began. We attribute these observations to a state-dependent effect on food consumption, since drug withdrawal could not account for the results. Both the SM-DM and MD-DM rats of the drugged fed group ate less than did DM-SM rats during early maze training, and they did not differ from each other, even though the MD-DM rats continued to receive injections of drug. However, as an additional check on the possibility of drug withdrawal, the postdrugged fed animals were included. Comparisons showed that food consumption was less for DMSM rats in the drugged fed group than for DM-SM rats in the postdrugged fed group, rats that had had equal drug experience beyond 28 days, and this points to an effect specific to feeding in the drugged state rather than to an effect of drug experience alone.

There were no reductions in food intake for the non-predrugged group, which showed that a period of "drugged-state feeding" longer than 28 days might be necessary to produce the dissociation effect; the duration of drugged-state feeding required remains undetermined.

There are three prominent theories of statedependent learning that could be applied to the maze training data of this experiment. First, the complexmediating hypothesis (Overton, 1964) is that cell assemblies mediate learned behaviors and these assemblies are altered by drugged states. During statedependent dissociation, the assemblies that were formed to mediate behavior learned in one state suddenly are discontinued and new cell assemblies (new learning) must be formed. Generalization to the new cell assemblies is made rapidly, according to the theory, and so the dissociation effect is considered to be a transient one. A second theory of state-dependent learning that might explain the maze training data is the simple-complex hypothesis advanced by Sachs (1967). This hypothesis is that the drugged state impairs the cognitive functioning of the animal such that the animal does not attend to many features of its environment. When the animal is not drugged after learning in the drugged state, it is flooded with those "novel" features of the environment to which it did not respond while drugged. Exploratory responses then are elicited which compete and interfere with the learned response. According to this theory, the poor performance of the SM-DM drugged fed rats during initial maze training and of the DM-SM drugged fed rats during maze retraining would occur because of exploratory reactions when these animals first encountered the maze in the nondrugged state. A third interpretation of state-dependent learning comes from the drug-as-stimulus hypothesis (Brown, Feldman, \& Moore,
1968; Otis, 1964; Overton, 1972). This position is that the drugged state has stimulus characteristics that are on a continuum with the nondrugged state, and that the drugged state acts as a discriminative stimulus that exerts control over the response. According to this interpretation, any reduction in feeding or increase in maze latency when the drug conditions were reversed would reflect a loss of the stimulus (drugged state) that signaled food. Hence, the animals in the present experiment would not have run the maze or eaten normally because the drugged state that signaled food was not present.

The problem with each of these interpretations is that none of them can explain the long-term disruptions of maze running of the DM-SM animals in each of the three main groups during maze retraining when drug conditions were reversed. During maze training, the performance of all groups had stabilized by the end of the 4th day. It should be expected, therefore, that performance during the retraining phase, when the animals were not drugged, also should have stabilized within the first 4 days. However, throughout maze retraining these groups had erratic performances characterized sometimes by short but mostly by long latencies. Furthermore, the latencies did not appear to be related to food consumption; that is, they remained erratic despite the fact that some animals resumed normal eating patterns immediately and all animals did so after 4 to 6 days. Thus, during maze retraining, the latencies no longer appeared to be related to the amount of food eaten.

According to the complex-mediating hypothesis, the DM-SM animals should have learned the maze utilizing one set of cell assemblies while drugged and another set while not drugged, and there should have been generalization across cell assemblies by the end of the first 4 days of retraining, at least by the end of 2 weeks. The simple-complex hypothesis also cannot explain the retraining data of the DM-SM animals, since the exploratory responses and novelty cues should have habituated at least as fast as they did during maze training. These animals were not drugged and should have adapted quickly to novelty cues in such a simple maze. The same argument exists against the drug-stimulus hypothesis. For SM-DM animals in each main group, the nondrugged state plus maze cues signaled food at least by the end of the first 4 days regardless of whether they had previous experience with druggedstate feeding. It is reasonable to expect DM-SM animals also to begin using maze cues or the nondrugged state to signal food after 4 days of experience in the nondrugged condition during maze retraining; but they did not. For DM-SM rats in both drugged fed and postdrugged fed groups, not only did maze cues fail to signal food, but the presence of food itself was not an adequate stimulus to begin eating. This was true even though the rats had fed previously in a nondrugged state, were 
used to scheduled feeding, had fed previously in the goalbox, were eating a familiar food, and were not ill from drug withdrawal.

It is especially difficult to interpret the current failures of running and eating as evidence of failure to attend to maze or state cues (the drug-stimulus hypothesis), since the rats initially failed even to attend to the goal object that the maze cues were supposed to signal. There was no need for cues to signal the presence or absence of a goal object when the animal already was in contact with it; stimulus cuing, here, should be irrelevant. But more importantly, the rats should have begun to utilize maze cues as signals for food soon after they began to show an interest in eating. The fact that they did not, were not drugged, and were in a relatively simple maze is suggestive of a disruption of functioning unrelated to stimulus cuing.

We originally proposed (Gill \& Nielson, Note 1) that Bindra's $(1969,1974)$ theoretical position could account for the present results. We no longer believe this to be true, since food presented to a food-deprived animal should have evoked feeding given the conditions of the present experiment. Instead, we now believe that the results can be interpreted best as representing a drug-induced loss of encephalized control over the behavior of the animals. That is, the drugged animals may have reverted to sensory-motor-integrated activity, to guide their feeding and running behavior, that was less complex in form. This concept is analogous to the concept of sensory neglect described by Teitelbaum and his colleagues (Marshall, Turner, \& Teitelbaum, 1971; Wolgin, Cytawa, \& Teitelbaum, 1976) for animals that were given lateral hypothalamic lesions and did not eat or drink. Basically the phenomenon of sensory neglect is that adipsic and aphagic lateral hypothalamic animals do not orient towards visual, olfactory, auditory, or tactile stimulation. However, the deficit does not appear to be strictly sensory or motor, since the animals appear capable of sensing stimulation, as indicated by eye movements and respiratory changes, and can perform normal grooming responses. Rather, the lateral hypothalamic animal seems unable to integrate sensory information with the appropriate motor sequences.

Recovery from the lateral hypothalamic syndrome, created either by brain lesions or by 6-hydroxydopamine (Marshall, Richardson, \& Teitelbaum, 1974), occurs over a long period of time with feeding and drinking preceding complex-sensory-integrated behavior. This recovery of function is reported to be similar to the normal sequence of infant-rat development where there is a period of encephalization of performance across time (Teitelbaum, Cheng, \& Rozin, 1969). We believe that the current findings are representative of a similar sequence; specifically, the drug possibly served to partially deencephalize complex integrated behavior, requiring basic feeding responses to be reestablished at a more reflexive level of integration. Then, when the drug was withheld, it took time for the feeding responses to become reencephalized, and it took even longer for any complex sensory-guided responses to begin to occur, if they occurred at all. Unfortunately, the experiment was not guided by this interpretation when it was being conducted or we would have carried out the procedures over a longer period of time and, perhaps, discovered the relative permanence of this effect.

The argument that our results and perhaps statedependent learning can be interpreted best in the framework of a drug-induced loss of sensary-motor-integrated activity, possibly similar to the effects of lateral hypothalamic lesions, hinges on observing the differences in recovery described above, but also on the argument that state-dependent learning may represent a failure of sensory-motor-integrated behavior analogous to sensory neglect. Considerable evidence has accumulated in recent years that drugged animals, while not blind or deaf, do not effectively use visual or auditory cues in discrimination tasks (Gross \& Weiskrantz, 1961; Weiskrantz \& Baltzer, 1965) and cannot use place cues to learn a water maze (Pusakulich \& Nielson, 1976). In fact, Pusakulich and Nielson have argued that drugs impair the ability of an animal to use environmental cues so that the animal must resort to a strategy of learning response sequences when drugged. A further restriction on the functioning of drugged animals has been explored by DeWitt and Nielson (Note 2), who conditioned cats to give foreleg-flexion conditioned responses simulaneously to a tone and to direct electrical stimulation of the brain, both as conditioned stimuli. Then, when the drug was administered, the conditioned responses to the tone were abolished at drug doses that left the same conditioned response intact, in the same animal, when the conditioned stimulus was direct electrical stimulation of the brain. Thus, the sensory conditioned stimulus could not maintain the conditioned response under the drugged condition. At the same time, there was no motor impairment that could explain the loss of the response, because the response was intact, at the same drug dosage level, when the conditioned stimulus was direct electrical stimuiation of the brain. Subsequent sensory tests of these cats showed that they did respond to dogs, mice, rats, and a variety of other stimulation. Thus it appears that drugs that produce state-dependent learning do so, not by any direct interference with either sensory or motor processes, but by interfering with their integration. It is this proposed interference with sensory-motor integrations that we believe makes state-dependent learning a consequence not of any failure of the drug to serve as a stimulus when the drugged state is altered, but of a change in central nervous system functioning where complex, encephalized sensory-motor-integrated performances are abolished. The animal neglects stimuli to which he attended while not drugged, and even normal feeding behavior 
can be disrupted if feeding has been limited to the drugged state for a period of about 45 days. It was the momentary loss of feeding, where a loss of a cue seems irrelevant, plus the relatively prolonged disruptions in maze running which lead us to think that the deficits commonly observed in state-dependency research may represent motivational deficits along a continuum of disrupted sensory-motor-integrated functioning, functioning that recovers differentially across time, rather than a product of discontinued cell assemblies, of a novel sensory environment, or of stimulus-cuing failure.

\section{REFERENCE NOTES}

1. Gill, J. H., \& Nielson, H. C. State-dependence of feeding and maze performance in rats. Paper presented at the meeting of the American Psychological Association, Chicago, August 1975.

2. DeWitt, J. R., \& Nielson, H. C. Differential effects of anesthetics on cue use in cats. In preparation.

\section{REFERENCES}

BINDRA, D. The interrelated mechanisms of reinforcement and motivation, and the nature of their influence on response. In M. J. Arnold \& D. Levin (Eds.), Nebraska Symposium on Motivation (Vol. 17). Lincoln: University of Nebraska Press, 1969.

BINDRA, D. A motivational view of learning, performance, and behavior modification. Psychological Review, 1974, 81, 199-213.

Bindra, D., Nyman, K., \& Wise, J. Barbiturate-induced dissociation of acquisition and extinction: Role of movement initiation processes. Journal of Comparative and Physiological Psychology, 1965, 60, 223-228.

BINDRA, D., \& REICHERT, H. Dissociation of movement initiation without dissociation of response choice. Psychonomic Science, 1966, 4, 95-96.

BINDRA, D., \& REICHERT, H. The nature of dissociation: Effects of transitions between normal and barbiturate-induced states on reversal learning and habituation. Psychopharmacologia, 1967, 10, 330.

Brown, A., Feldman, R. S., \& Moore, J. W. Conditional discrimination learning based on chlordiazepoxide: Dissociation or cue? Journal of Comparative and Physiological Psychology, 1968, 66, 211-215.

Collier, H. O. J. Humoral transmitters, supersensitivity, receptors and dependence. In H. Steinberg (Ed.), Scientific basis of drug dependence: $A$ symposium. New York: Grune and Stratton, 1969.

DeVietti, R. L., \& Larson, R. C. ECS effects: Evidence supporting state-dependent learning in rats. Journal of Comparative and Physiological Psychology, 1971, 74, 407-415.

Girden, E., \& Culler, E. A. Conditioned responses in curarized striate muscle in dogs. Journal of Comparative and Physiological Psychology, 1937, 23, 261-274.

Goodwin, D. W., Powell, B., Bremer, D., Hoine, H., \& STERN, J. Alcohol and recall: State dependent effects in man. Science, 1969, 163, 1358-1360.

Gross, C. G., \& Weiskrantz, L. The effect of two "tranquilizers" on auditory discrimination and delayed response performance of monkeys. Quarterly Journal of Studies on Alcohol, 1961, 13, 34-37.

Holmgren, S. Conditional avoidance reflex under pentobarbital. Boletin del Instituo de Estudio Medicos y Biologics (Mexico), 1964, 22, 21-37.

KubenA, R. K., \& BARRY, H., III. Generalization by rats of alcohol and atropine stimulus characteristics to other drugs. Psychopharmacologia (Berlin), 1969, 15, 196-286.

Marshall, J. F., Richardson, J. S., \& Teitelbaum, P. Nigrostriatal bundle damage and the lateral hypothalamic syndrome. Journal of Comparative and Physiological Psychology, 1974, 87, 808-830.

Marshall, J. F., Turner, B. H., \& Teitelbaum, P. Sensory neglect produced by lateral hypothalamic damage. Science, $1971,174,523-525$.

Madill, M. Alcohol induced dissociation in humans: A possible treatment technique for alcoholism. Unpublished doctoral dissertation, Queens University, Kingston, Ontario, Canada, 1967.

Nielson, H. C. Evidence that electroconvulsive shock alters memory retrieval rather than memory consolidation. Experimental Neurology, 1968, 20, 3-20.

OTis, L. S. Dissociation and recovery of a response learned under the influence of chlorpromazine or saline. Science, 1964, 143, 1347.

Overton, D. A. State-dependent or "dissociated" learning produced with pentobarbital. Journal of Comparative and Physiological Psychology, 1964, 57, 3-12.

Overton, D. A. Stimulus control of behavior by drug states. In T. Thompson \& R. Pickens (Eds.), Stimulus properties of drugs. New York: Appleton-Century-Crofts, 1971. Pp. 87-110.

Overton, D. A. Experimental methods for the study of statedependent learning. Federation Proceedings, 1974, 33, 1800-1813.

Pusakulich, R. L., \& Nielson, H. C. Neural thresholds and state-dependent learning. Experimental Neurology, 1972, 34. 33-34.

Pusakulich, R. L., \& Nielson, H. C. Cue use in state-dependent learning. Physiological Psychology, 1976, 4, 421-428.

Robbins, M. J., \& MeYer, D. R. Motivational control of retrograde amnesia. Journal of Experimental Psychology, 1970, 84, 220-225.

SACHS, E. Dissociation of learning in rats and its similarities to dissociative states in man. In J. Zubin \& H. F. Hunt (Eds.), Comparative psychopathology: Animal and human. New York: Grune and Stratton, 1967.

Swanson, J. M., \& Kinsbourne, M. Stimulant-related statedependent learning in hyperactive children. Science, 1976, 192, 1354-1357.

Teitelbaum, P., Cheng, M. F., \& Rozin, P. Development of feeding parallels its recovery after hypothalamic damage. Journal of Comparative and Physiological Psychology, 1969, 67, 430-441.

WEISKRANTZ, L., \& BALTZER, V. A note on further experiments of meprobamate on discrimination performance in the monkey. Quarterly Journal of Experimental Psychology, 1965, 17, 169-172.

WIKLER, A. Opate addiction. Springfield, Ill: Thomas, 1953.

Wolgin, D. L. Cytawa, J., \& Teitelbaum, P. The role of activation in the regulation of food intake. In D. Novin, W. Wyrwicka, \& G. Bray (Eds.;, Hunger: Basic mechanisms and clinical implications. New York: Raven Press, 1976.

(Received for publication May 23, 1977; revision accepted February 2,1978 .) 